\section{Fasciitis in Mixed Connective Tissue Disease Successfully Treated with High-dose Intra- venous Immunoglobulin}

Key words: creatine kinase, muscle biopsy, diffuse fasciitis with eosinophilia, magnetic resonance imaging

A 36-year-old woman was admitted to our hospital in January 2000 because of sudden-onset fever and myalgia. Physical examination showed proximal muscle weakness without skin lesions, and laboratory data demonstrated an increase in serum creatine kinase $(\mathrm{CK}, 5,561 \mathrm{U} / l$, normal value less than $165 \mathrm{U} / l$ ) and inflammatory markers. Muscle biopsy showed perivascular infiltration of mononuclear cells, leading to a diagnosis of polymyositis. Anti-nuclear and anti-U1ribonucleoprotein (RNP) antibodies were negative, and an intensive systemic survey could not detect any malignancy. Since the patient weighed $80 \mathrm{~kg}$, oral prednisolone was started at a dose of $80 \mathrm{mg} /$ day $(1 \mathrm{mg} / \mathrm{kg} /$ day $)$. Her symptoms improved in parallel with a decrease in serum CK and inflammatory markers. At our outpatient clinic the daily dose of prednisolone was gradually tapered off to $12.5 \mathrm{mg}$ /day, due to bilateral glaucoma, hypertension, obesity and pneumonia associated with corticosteroid therapy.
In August 2001, the patient again developed fever and myalgia mainly in the bilateral thighs, but this time the serum CK level was normal. Despite additional administration of non-steroidal anti-inflammatory drugs her symptoms gradually worsened until she became unable to walk and she was eventually readmitted to our hospital in November. Physical examination found mild edema of bilateral hands as well as acrosclerosis, Raynaud's phenomenon and severe grasping pain in the bilateral thighs. Routine laboratory data showed no abnormal findings, including CK (125 U/l), except for an increased level of CRP $(2.8 \mathrm{mg} / \mathrm{dl}$, normal value less than $0.1 \mathrm{mg} / \mathrm{dl})$ and $\mathrm{WBC}\left(15,300 / \mathrm{mm}^{3}\right.$, normal $3,500 \sim 9,800 / \mathrm{mm}^{3}$ ), while no eosinophilia was detected by WBC differentiation. Anti-nuclear and anti-U1-RNP antibodies showed high titers $(\times 1,280$ and more than $\times 16$, respectively), but other autoantibodies, including anti-Sm, were all negative. ${ }^{67} \mathrm{Ga}$-citrate scintigraphy showed a positive shadow in the proximal portions of the bilateral extremities, particularly in the thighs (Fig. 1A). Magnetic resonance imaging (MRI) demonstrated thickening of muscle fascia in the bilateral thighs with marked enhancement of gadolinium (Fig. 1B). Biopsy obtained from the biceps femoris demonstrated gross thickening and a white appearance of the fascia, while histopathologic findings indicated mild infiltration of mononuclear cells into the fascia as well as muscles (Fig. 1C). Informed consent for the use of immunosuppressants

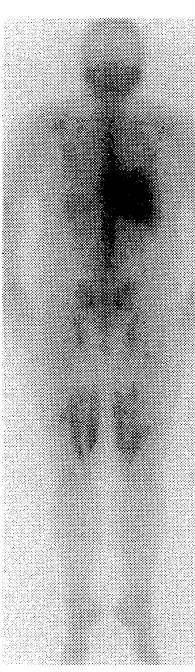

A

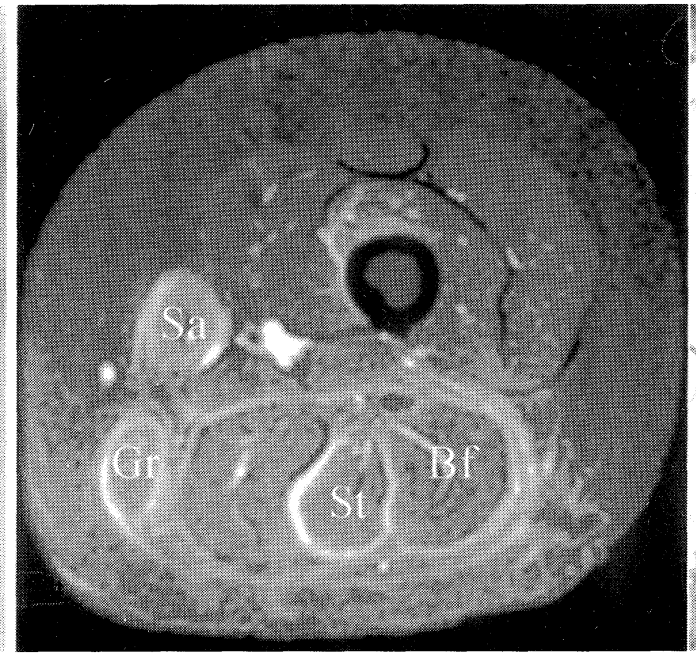

$\mathrm{B}$

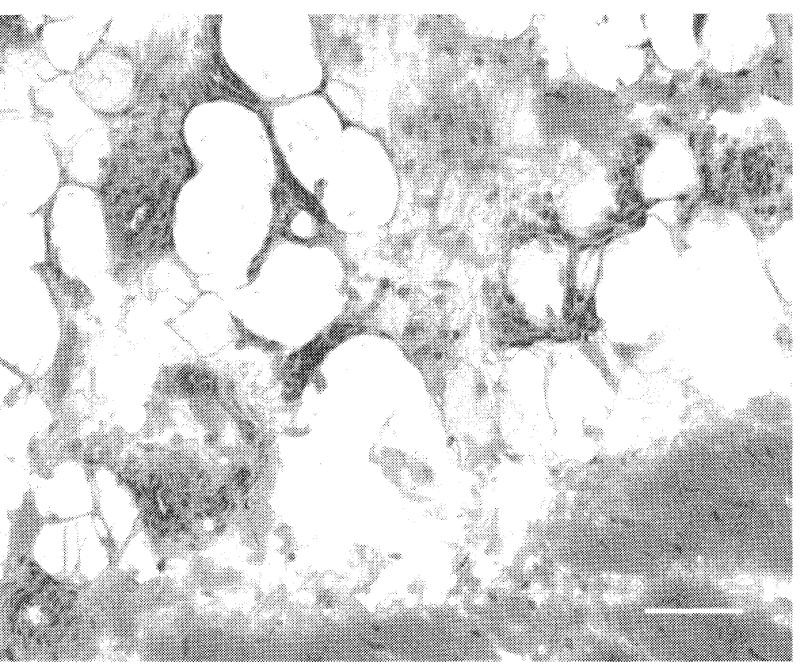

C

Figure 1. Whole body scintigraphy using ${ }^{67}$ Ga-citrate (A) showed an apparently positive shadow in the proximal portion of the extremities, particularly in the bilateral thighs. Magnetic resonance imaging demonstrated thickening of muscle fascia in the right thigh, particularly in the long head of biceps femoris (Bf), semitendinosus (St), gracilis (Gr) and sartorius (Sa), accompanied by marked enhancement of gadolinium ( $B, T R=500, T E=8.8)$. Histopathology of the biopsied muscle fascia showed interstitial edema with mild infiltration of mononuclear cells mainly in perivascular areas $(\mathrm{C}, \mathrm{HE}$ stain, bar=60 $\mu \mathrm{m})$. 
could not be obtained from the patient, so that high-dose intravenous immunoglobulin (IVIG) therapy was administered at a dose of $0.4 \mathrm{~g} / \mathrm{kg} /$ day for 5 days. In response to this therapy the symptoms quickly improved in parallel with a decrease of CRP. The patient left our hospital at the end of December, and she has since been maintained on around 10 $\mathrm{mg} /$ day of oral prednisolone. She has remained in good general condition without any muscular symptoms.

When severe myalgia reappeared mainly in bilateral thighs, exacerbation of polymyositis was at first seen as a possible diagnosis, but radiological and biopsy findings led to an eventual diagnosis of fasciitis. There are several general causes of fasciitis $(1,2)$, and the criteria proposed by the Ministry of Health and Welfare of Japan (3) led to a strong suspicion of an autoimmune mechanism because MCTD was thought to be present in the background. In fact, since the fasciitis developed in conjunction with the appearance of anti-U1-RNP antibody, MCTD itself may have played an important role in the pathogenesis. Recently, IVIG has been tried in a refractory case with MCTD (4), and this therapy was also successfully used for the treatment of fasciitis in our patient. In general IVIG acts on various immunomodulatory mechanisms, including inhibition of complement binding, downregulation of antibody production and neutralization of pathogenic cytokines (5). IVIG may thus be a therapeutic option for atypical cases with MCTD as in the present patient, in spite of the high cost, particularly when neither corticosteroid nor immunosuppressants can be used.

$$
\begin{array}{r}
\text { Masayuki MatsudA*,**, Jun MikI*, Kenya OGUCHI*, } \\
\text { Kenichi TABATA* and Shu-ichi IKEDA** }
\end{array}
$$

*The Department of Neurology, Saku Central Hospital, Usuda and **the Third Department of Medicine, Shinshu University School of Medicine, Matsumoto

Received for publication February 28, 2003; Accepted for publication June 13, 2003

Reprint requests should be addressed to Dr. Masayuki Matsuda, the Third Department of Medicine, Shinshu University School of Medicine, 3-1-1 Asahi, Matsumoto 390-8621

\section{References}

1) Wigley FM. Systemic sclerosis. Clinical features. in: Rheumatology. 2nd ed. Klippel JH, Dieppe PA, Eds. Mosby, London, 1998: 7.9.1-14.

2) Hübscher O. Pattern recognition in arthritis. in: Rheumatology. 2nd ed. Klippel JH, Dieppe PA, Eds. Mosby, London, 1998: 2.3.1-6.

3) Kasukawa R, Tojo T, Miyawaki S, et al. Preliminary diagnostic criteria for classification of mixed connective tissue disease. in: Mixed connective tissue disease and antinuclear antibodies. Kasukawa R, Sharp GC, Eds. Excerpta Medica, Amsterdam, 1987: 41-47.

4) Ulmer A, Kotter I, Pfaff A, Fierlbeck G. Efficacy of pulsed intravenous immunoglobulin therapy in mixed connective tissue disease. J Am Acad Dermatol 46: 123-127, 2002.

5) Dalakas MC. Mechanism of action of intravenous immunoglobulin and therapeutic considerations in the treatment of autoimmune neurologic diseases. Neurology 51 (Suppl): S2-8, 1998. 